\title{
Chaotic Characteristic Analysis of Brushless DC Motor with Vibration Load Disturbance
}

\author{
Jun Li ${ }^{1{ }^{* *}, \text { Libiao Wang }}{ }^{1}$, Jing Shi ${ }^{2}$ and Yongchao Zhang ${ }^{1}$ \\ ${ }^{1}$ College of Aeronautical Engineering, Taizhou University, Taizhou, Zhejiang, 318000, China \\ ${ }^{2}$ Department of Mechanical and Materials Engineering, University of Cincinnati, OH 45221, United States
}

Received 11 September 2018; Accepted 20 December 2018

\begin{abstract}
A brushless DC motor (BLDCM) is a kind of nonlinear system with multivariable coupling. Under certain conditions, chaos and other non-linear dynamic phenomena will occur and bring adverse effects to the motor itself and the drive system. However, in existing research on chaotic mechanism of BLDCM, the chaotic phenomena caused by the disturbance of load torques are seldom involved. However, in practical engineering applications, the disturbance in the load of the motor is extensive. To analyze the non-linear characteristics of the BLDCM caused by this disturbance, a disturbance variable was added to the $\mathrm{d}$-q axis mathematical model of the BLDCM. Then, a non-autonomous system model of 5-D BLDCM was constructed by using time scale and linear affine transformations. First, the non-linear dynamic characteristics of the system caused by the torque disturbance were analyzed by calculating the dissipation and equilibrium points of the system. Second, the chaotic dynamic behavior of the system under load disturbance was discussed through Lyapunov exponent and bifurcation graph. Finally, the accuracy of the model and analysis was verified through experiments. Results show that when the frequency of the disturbance component of the load increases from 0 $\mathrm{Hz}$ to $100 \mathrm{~Hz}$, the system experiences various motions, such as stable, period doubling, paroxysmal chaos, and periodic. This study provides a theoretical reference for further exploring the engineering physical characteristics of the BLDCM under load disturbance and searching for an improved control method.
\end{abstract}

Keywords: Brushless DC motor, Bifurcation, Chaos, Nonlinear, Vibration load

\section{Introduction}

A brushless DC motor (BLDCM) has been extensively used in the fields of robotics, precision machine tool transmission, aerospace, and automobile drive given its advantages, such as simple structure, reliable operation, high efficiency, and low electromagnetic interference [1]. With the popularization of the BLDCM, high requirements are proposed for improved performance. Among these requirements, the control accuracy of speed and torque is an important index. Nonlinear characteristics of the BLDCM are the primary factors that affect the precise control of the speed and torque of this system [2-3] Chaos is a typical manifestation of the nonlinearity for BLDCM. The occurrence of chaotic phenomena in the motor causes violent oscillations of speed and torque, seriously affects the stability and reliability of the system, and even triggers unexpected accidents [4].

At present, research on the chaotic behavior of the BLDCM has primarily focused on the chaotic phenomenon caused by the parameter change in the motor itself or control input and has seldom involved the chaotic behavior caused by torque disturbance [5-7]. Therefore, exploring the mechanism of chaos caused by load disturbance and analyzing the conditions of chaos formation are theoretical innovations of the present study. At present, research on

*E-mail address: tzxylijun@126.com

ISSN: $1791-2377$ @ 2018 Eastern Macedonia and Thrace Institute of Technology. All rights reserved.

doi:10.25103/jestr.116.05 non-linear behavior of a BLDCM is mainly based on the theory analysis and simulation of a mathematical model; moreover, experimental verification is rarely applied[8-9], because the actual chaotic state is difficult to maintain and observe; furthermore, the conditions for entering the chaotic state are special.

On the basis of the abovementioned reasons, the present study focuses on the nonlinear characteristics of the BLDCM caused by load torque vibration. The relationship between the frequency of torque vibration and the non-linear behavior of the motor is analyzed by solving the mathematical model of the motor and simulation. Simultaneously, the theoretical model and analysis are verified by experiments.

\section{State of the Art}

The BLDCM is a multivariable coupled nonlinear system. On certain conditions, the system will produce complex nonlinear dynamic behavior [10]. By analyzing a dimensionless BLDCM mathematical model, Hemati [11] initially found a chaotic motion in the system. Hemati's research showed that, when the input of the system is constant and the viscosity coefficient is a certain value, the phase trajectory of the motor presents a singular attractor behavior.

Zhang et al. [8] used Lyapunov stability theory to analyze a 3D autonomous system model of the BLDCM; 
these authors verified that the chaotic motion of the system is bounded; they also calculated the switching rate of the system phase trajectory among different attractors. Farzaneh et al. [12] studied the chaos of the BLDCM model with a single time-scale transformation. System simulation showed that, when the system parameters enter a specific region, the BLDCM system enters into a chaotic motion and uses an inverse control to synchronize the chaos. Mousam et al. [13] analyzed the nonlinear characteristics of a permanentmagnet BLDCM; these researchers discussed the chaos, bifurcation and other non-linear phenomena caused by slot structure and power transistor switching. Tahir et al. [14] discussed the nonlinear characteristics of a permanentmagnet DC motor driven by PID control; these authors reported periodic, quasi-periodic, and chaotic behaviors in the motor and used a sliding mode method to control chaos. In accordance with the mathematical model of a permanentmagnet brushless motor, $\mathrm{Hu}$ et al. [15] confirmed the existence of chaos in the system through an equilibrium point and phase plane analysis method; these researchers used a robust nonlinear control to achieve speed stability under torque disturbance. However, the chaos in the analysis was only caused by the internal characteristics of the motor and not by the load disturbance. The abovementioned study on the nonlinearity of the BLDCM primarily focused on the chaotic phenomenon caused by the parameter change in the motor itself or the controller. It also ignored the chaotic behavior caused by the vibration disturbance of the motor load. However, in practical engineering applications, vibration loads are ubiquitous; furthermore, the resulting nonlinear motion of the system has a general impact on the stability of the system.

Hemati et al. [16] established a mathematical model of the BLDCM considering a nonuniform air gap and magnetic saturation. Scholars have studied the nonlinear phenomenon of the BLDCM on the basis of this model. Sukanya [6] used a $3 \mathrm{D}$ mathematical model of the BLDCM to analyze the influence of controller gain, torque, and speed on the nonlinear characteristics of the motor, these researcher derived the corresponding domain of periodic and chaotic motions. Based on the 3-D d-q axis model of the BLDCM, Qian et al. [17] developed the torque ripple equation caused by switching action of power transistor using a 3-D BLDCM mathematical model. Yoni et al. [18] discussed the mathematical equation of torque ripple generated by the back EMF of the motor and used an adaptive internal model control to suppress the torque ripple. Tian et al. [19] used a 3-D BLDCM mathematical model to analyze the nonlinear characteristics of the motor and used a feedback control to achieve global stability. Shahri et al. [20] analyzed the stability of a fractional order system and used state feedback to realize the stability of the system. The control method was applied to the BLDCM. However, the fractional order model only involves three parameters, namely, straight axis current, quadrature axis current, and angular velocity; this model does not involve the motor torque. On the basis of Hemati's model of the 3-D BLDCM, Yilmaz et al. [21] proposed a sliding mode control method for realizing the chaotic control of motor. The abovementioned mathematical models used to analyze the chaotic behavior of the BLDCM are all 3D mathematical models that do not take load torque as one variable for establishing a high-dimensional motor mathematical model.

On the basis of the abovementioned problems, the present study analyzed the nonlinear phenomena of the BLDCM, such as chaos caused by load vibration, and used a load torque as a variable. A 5-D dimensionless mathematical model based on 3-D d-q axis model was proposed to perform a theoretical analysis. To verify the accuracy of the theoretical model and simulation analysis, an experimental platform was built.

The remainder of this study is organized as follows. Section 3 presents a 5-D BLDCM mathematical model with vibration load disturbance. The nonlinear characteristics of the model are analyzed theoretically, simulated, and verified. Section 4 analyzes the simulation and experimental results. Section 5 summarizes the conclusions drawn from this study.

\section{Methodology}

\subsection{Mathematical model of the BLDCM under vibration load disturbance}

The BLDCM is a multivariable coupled nonlinear system. Through coordinate transformation, the mathematical model of this scheme in the $\mathrm{d}-\mathrm{q}$ coordinate system can be described as [22].

$$
\left\{\begin{array}{l}
\frac{d i_{d}}{d t}=\frac{1}{L_{d}}\left(u_{d}-R i_{d}+n_{p} L_{q} \omega i_{q}\right) \\
\frac{d i_{q}}{d t}=\frac{1}{L_{q}}\left(u_{q}-R i_{q}-n_{p} L_{d} \omega i_{d}-n_{p} k_{t} \omega\right) \\
\frac{d \omega}{d t}=\frac{1}{J}\left(n_{p} k_{t} \omega i_{q}+n_{p}\left(L_{d}-L_{q}\right) i_{d} i_{q}-\beta \omega-T_{L}\right)
\end{array}\right.
$$

where $u_{d}, i_{d}, u_{q}$ and $i_{q}$ denote the direct axis voltage, direct axis current, quadrature axis voltage, and current of the motor, respectively. $L_{d}$ and $L_{q}$ represent the direct and quadrature axis inductance, correspondingly; $\mathrm{R}$ is the stator resistor. $k_{e}$ is the permanent magnet flux linkage. $k_{t}=\sqrt{3 / 2} k_{e}, \omega, n_{p}$ are the angular velocity and the pole pair number of the motor. $J$ and $\beta$ are the moment of inertia and the viscosity coefficient of the motor. $T_{L}$ is the load torque of the motor.

In this study, we simulate the actual vibration load with sinusoidal function. The vibration load is expressed as

$$
T_{L}=B+A \cos \left(\omega t+\varphi_{0}\right)
$$

where $\mathrm{B}$ is the constant component of load, $\mathrm{A}$ is the amplitude of vibration load, $\omega=2 \pi f, f$ is the frequency of the disturbance component of load torque, and $\varphi_{0}$ is the initial phase of the disturbance component.

According to Equations (1) and (2), when the BLDCM system is disturbed by the vibration load, the equation of the system contains the time variable $t$. Thus, the system is a non-autonomous system. For the convenience of the nonlinear analysis of the system, we use

$u=\cos \left(\omega t+\varphi_{0}\right)$ and $v=\sin \left(\omega t+\varphi_{0}\right)$

The system is then transformed into an autonomous system. To establish a dimensionless model of the BLDCM through linear affine and time scale transformations, 
let $\mathrm{x}=\sigma \tilde{\mathrm{x}}$ and $t=\tau \tilde{t}$

where

$$
\begin{aligned}
& \mathrm{x}=\left[x_{1}, x_{2}, x_{3}, x_{4}, x_{5}\right]^{\mathrm{T}}=\left[i_{d}, i_{q}, \omega, u, v\right]^{\mathrm{T}}, \\
& \tilde{\mathrm{x}}=\left[\tilde{x}_{1}, \tilde{x}_{2}, \tilde{x}_{3}, \tilde{x}_{4}, \tilde{x}_{5}\right]^{\mathrm{T}}=\left[\tilde{i}_{d}, \tilde{i}_{q}, \tilde{\omega}, \tilde{u}, \tilde{v}\right]^{\mathrm{T}},
\end{aligned}
$$$$
\tau=\frac{L_{q}}{R}, \sigma=\left[\begin{array}{lllll}
\sigma_{1} & 0 & 0 & 0 & 0 \\
0 & \sigma_{2} & 0 & 0 & 0 \\
0 & 0 & \sigma_{3} & 0 & 0 \\
0 & 0 & 0 & \sigma_{4} & 0 \\
0 & 0 & 0 & 0 & \sigma_{5}
\end{array}\right]
$$

and

$\sigma_{1}=\delta \sigma_{2}, \sigma_{2}=\frac{J \sigma_{2}^{3}}{\delta k_{t}}, \sigma_{3}=\frac{1}{n_{p} \tau}, \sigma_{4}=\sigma_{5}=1, \delta=\frac{L_{q}}{L_{d}}$

After linear transformation, the dimensionless model of the BLDCM under the disturbance of vibration load is presented as follows:

$$
\left\{\begin{array}{l}
\dot{\tilde{x}}_{1}=-\delta \tilde{x}_{1}+\tilde{x}_{2} \tilde{x}_{3}+\tilde{u}_{d} \\
\dot{\tilde{x}}_{2}=-\tilde{x}_{2}-\tilde{x}_{1} \tilde{x}_{3}-\rho \tilde{x}_{3}+\tilde{u}_{q} \\
\dot{\tilde{x}}_{3}=\tilde{x}_{2} / \delta+\eta \tilde{x}_{1} \tilde{x}_{2}-\gamma \tilde{x}_{3}-\mu A \tilde{x}_{4} \\
\dot{\tilde{x}}_{4}=-\omega B \tau \tilde{x}_{5} \\
\dot{\tilde{x}}_{5}=\omega \tau \tilde{x}_{4}
\end{array}\right.
$$

where

$$
\begin{aligned}
& \tilde{u}_{d}=\frac{\tau}{\sigma_{1} L_{d}} u_{d}, \tilde{u}_{q}=\frac{\tau}{\sigma_{2} L_{q}} u_{q}, \rho=\frac{k_{t}}{\sigma_{2} L_{q}}, \\
& \eta=\frac{\left(L_{d}-L_{q}\right) \sigma_{1} \sigma_{2}}{J}, \gamma=\frac{\beta \tau}{J}, \mu=\frac{\tau}{\sigma_{3} J}
\end{aligned}
$$

When the magnetic leakage effect of the air gap is ignored, the motor parameters are provided as follows: rated power $p_{N}=1.5 \mathrm{kw}$, rated speed $n_{N}=3000 \mathrm{r} / \mathrm{min}$, rated torque $T_{N}=5 \mathrm{~N} \cdot \mathrm{m}$, inductance of $\mathrm{d}-\mathrm{q}$ axis $L_{d}=L_{q}=16.5 \mathrm{mH}$, stator resistance $\mathrm{R}=0.37 \Omega$, rotating inertia $J=0.0012 \mathrm{~kg} \cdot \mathrm{m}^{2}$, viscosity coefficient $\beta=0.025 \mathrm{~kg} /(\mathrm{m} \cdot \mathrm{s})$, permanent magnet flux $k_{e}=0.031 \mathrm{wb}$, and pole pair number of motor $n_{p}=4$. The parameters of vibration torque are provided as follows: $A=1 \mathrm{~N} \cdot \mathrm{m}, \quad \mathrm{B}=3 \mathrm{~N} \cdot \mathrm{m}, \quad$ by substituting the abovementioned parameters of the motor into Equation (3), we determine

$$
\left\{\begin{array}{l}
\dot{\tilde{x}}_{1}=-\tilde{x}_{1}+\tilde{x}_{2} \tilde{x}_{3}+\tilde{u}_{d} \\
\dot{\tilde{x}}_{2}=-\tilde{x}_{2}-\tilde{x}_{1} \tilde{x}_{3}-2.362 \tilde{x}_{3}+\tilde{u}_{q} \\
\dot{\tilde{x}}_{3}=\tilde{x}_{2}-0.938 \tilde{x}_{3}-6.749 \tilde{x}_{4} \\
\dot{\tilde{x}}_{4}=-0.045 \omega \tilde{x}_{5} \\
\dot{\tilde{x}}_{5}=0.045 \omega \tilde{x}_{4}
\end{array}\right.
$$

\subsection{Dynamics analysis of the BLDCM under oscillating} load

To study the nonlinear dynamics of the BLDCM, the dissipation of the system must be analyzed. In accordance with Equation (4), the divergence of the system is

$$
\begin{aligned}
\nabla \cdot F & =\frac{\partial \dot{\tilde{x}}_{1}}{\tilde{x}_{1}}+\frac{\partial \dot{\tilde{x}}_{2}}{\tilde{x}_{2}}+\frac{\partial \dot{\tilde{x}_{3}}}{\tilde{x}_{3}}+\frac{\partial \dot{\tilde{x}}_{4}}{\tilde{x}_{4}}+\frac{\partial \dot{\tilde{x}}_{5}}{\tilde{x}_{5}} \\
& =-1-1-0.938+0+0=-2.938<0
\end{aligned}
$$

The divergence of the system is less than zero. As such, the system is a negative source field. When $t \rightarrow \infty$, each volume element that contains the phase trajectories of the system will shrink to zero at an exponential rate $e^{-2.938}$, that is, all phase trajectories of the system are limited to a set of zero volume elements. Therefore, the system has the attractor.

\subsection{Stability analysis of equilibrium point}

The dynamic characteristics of the equilibrium point directly affect the dynamic characteristics of the system. In accordance with Equation (4), the nonlinear algebraic equation for calculating an equilibrium point can be written as

$\left\{\begin{array}{l}-\tilde{x}_{1}+\tilde{x}_{2} \tilde{x}_{3}+\tilde{u}_{d}=0 \\ -\tilde{x}_{2}-\tilde{x}_{1} \tilde{x}_{3}-2.362 \tilde{x}_{3}+\tilde{u}_{q}=0 \\ \tilde{x}_{2}-0.938 \tilde{x}_{3}-6.749 \tilde{x}_{4}=0 \\ -0.045 \omega \tilde{x}_{5}=0 \\ 0.045 \omega \tilde{x}_{4}=0\end{array}\right.$

By solving Equation (6), we can obtain two equilibrium points of the system as follows:

$\mathrm{s}_{1}(\mathrm{p}-1.65 \mathrm{q}+5.8,0.47 \mathrm{q}-1.65,0.5 \mathrm{q}-1.76,0,0)$

$s_{2}(p+1.65 q+5.8,-0.47 q-1.65,-0.5 q-1.76)$

where

$p=\tilde{u}_{d}+\tilde{u}_{q}, q=\sqrt{4.264 \tilde{u}_{q}+12.377}$

The stability of the equilibrium point of the system is analyzed in two situations:

1) $\tilde{u}_{d}=\tilde{u}_{q}=0$

This situation is equivalent to cutting off the external power supply when the motor is running, the motor is in an uncontrolled condition. Clearly, in this case, two equilibrium points of the system are 
$S_{1}^{0}=(0,0,0,0,0)$ and $S_{2}^{0}=(11.61,-3.3,-3.52,0,0)$.

Using Equation (4), the Jacobian matrix of the system can be obtained as follows:

$J a c=\left(\begin{array}{ccccc}-1 & \tilde{x}_{3} & \tilde{x}_{2} & 0 & 0 \\ -\tilde{x}_{3} & -1 & -2.362-\tilde{x}_{1} & 0 & 0 \\ 0 & 1 & -0.938 & -6.749 & 0 \\ 0 & 0 & 0 & 0 & -0.045 \omega \\ 0 & 0 & 0 & 0.045 \omega & 0\end{array}\right)$

Therefore, the eigenvalues that correspond to the two equilibrium points can be calculated. The eigenvalues that correspond to equilibrium point $s_{1}^{0}$ are

$\left\{\begin{array}{l}\lambda_{1}=-1 \\ \lambda_{2,3}=-0.97 \pm 1.54 i \\ \lambda_{4,5}= \pm 0.045 \omega i\end{array}\right.$

Then, the eigenvalues that correspond to equilibrium point $s_{2}^{0}$ are are shown in equation (9).

The eigenvalues that correspond to the two equilibrium points all have a pair of conjugate pure imaginary root. The remaining eigenvalues all have negative real parts. Therefore, the two equilibrium points are called the center point. In this situation, the system is stable. However, non-asymptotic stability is observed at the equilibrium point.

$\left\{\begin{array}{l}\lambda_{1}=-1.41 \\ \lambda_{2,3}= \pm 0.045 \omega i \\ \lambda_{4,5}=-0.76 \pm 5.15 i\end{array}\right.$

2) $\tilde{u}_{d} \neq 0, \tilde{u}_{q} \neq 0$

In this situation, the motor is under a normal working condition. The equilibrium points contain two variables $p$, $q$.According to Equation (7), the order of Jacobian matrix is 5. Thus, solving the eigenvalues that correspond to the equilibrium point is difficult. Nonetheless, two eigenvalues of the system can be solved by symbolic numerical solution $\lambda_{1,2}= \pm 0.045 \omega i$. The remaining three eigenvalues are polynomial real numbers or complex roots with three variables $p, q, \omega$.

The existence of a pair of pure imaginary eigenvalues is a necessary condition for Hopf bifurcation. The system has a pair of conjugate pure imaginary eigenvalues at the equilibrium point. Therefore, by adjusting the parameters of the three variables appropriately, the system can generate complex nonlinear dynamical behaviors near singularities, including bifurcation and chaos phenomena.

\subsection{Analysis of Lyapunov exponent and bifurcation diagram}

We use Lyapunov exponential and bifurcation trajectory varying with the frequency of oscillating load to describe the relationship between the dynamic characteristics of the system and the frequency of the oscillating load. On the basis of the stability analysis of the equilibrium point, complex non-linear phenomena will appear in the system if the relevant parameters are properly adjusted. We set the simulation parameters in accordance with the performance of the experimental motor. If the motor is controlled only by the $\mathrm{q}$ axis and the input is constant, then $\tilde{u}_{d}=35, \tilde{u}_{q}=35$,

and the initial phase of the load vibration $\varphi_{0}=0^{\circ}$.

When the frequency of the load vibration component varies from $0 \mathrm{~Hz}$ to $100 \mathrm{~Hz}$, the Lyapunov exponent of the system that corresponds to Equation (4) is depicted in Fig. 1. In this figure, LE1, LE2, LE3, LE4, and LE5 represent five Lyapunov exponents of the system. LE4 and LE5 graphics overlap. Thus, only LE4 is labeled in the figure. To analyze the relationship between the non-linear dynamic characteristics of the system and the frequency of the vibration load further, we design the Poincar-section $\left(\tilde{x}_{1}=0\right.$ ) and draw the bifurcation diagram of the system, as demonstrated in Fig. 2.

In accordance with the Lyapunov exponent method proposed by Chen et al., the dynamic characteristics of highdimensional systems can be judged [23]. By combining Fig. 1 and Fig. 2, the system obtains the following dynamic behaviors:

(1) When the frequency $f \in[0,6.6]$ (In $\mathrm{Hz}$, the following are the same), LE1,LE2,LE3,LE4 $<0$. That is, the phase trajectory of the system converges to a stable point.

(2) When the frequency $f \in[6.7,43.9],[52,54.2]$

[58.9,65],[66.4,67.7],[71,78],[84.2,100],

$\mathrm{LE} 1, \mathrm{LE} 2, \mathrm{LE} 3<0, \mathrm{LE} 4=0$. That is, the phase trajectory of the system runs in periodic orbits.

(3) When the frequency $f \in[43.9,52],[54.2$, 58.9],[65,66.4],[67.7,71] ,78,84.2],LE1 $>0, \quad$ LE2, LE3<0, LE4 $=0$. That is, the system is in a chaotic state, and a strange attractor exists. That is, with the change in the vibration frequency of the load torque, the phase trajectory of the motor has the characteristics of limit cycle, periodic motion, double periodic motion, and chaotic motion.

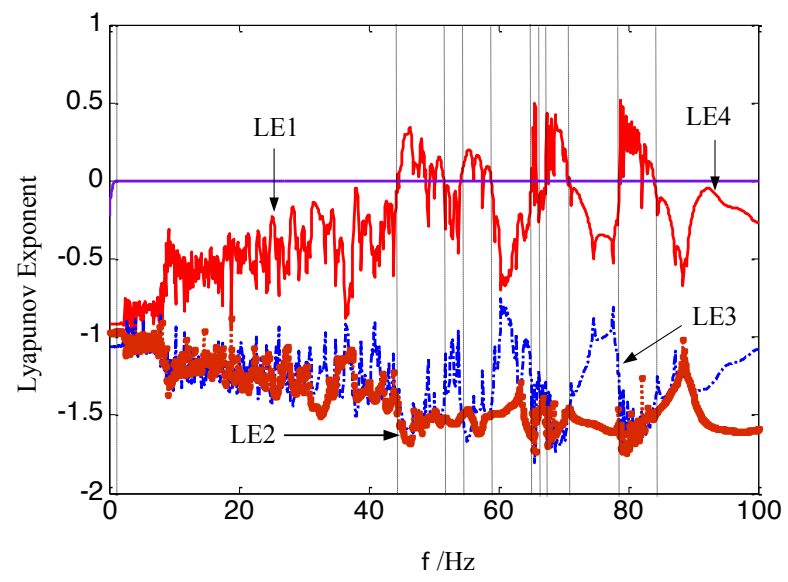

Fig. 1. Lyapunov exponent of the BLDCM

\subsection{Simulation study}

To verify the non-linear characteristics of the BLDCM under vibration load disturbance, we conduct simulation research. 
For the system of Equation (4), the initial state of the system is set as follows: $\left[\tilde{i}_{d}, \tilde{i}_{q}, \tilde{\omega}, \tilde{u}, \tilde{v}\right]^{\mathrm{T}}=[0.1,0.1,0.1,1,0.1]^{\mathrm{T}}$. The controlled input is set as follows: $\tilde{u}_{d}=0$, and $\tilde{u}_{q}=35$. When the frequency of the disturbance component of the motor torque increases gradually from $0 \mathrm{~Hz}$ to $100 \mathrm{~Hz}$, four motion modes appear in the system simulation (Fig. $3 \sim$ Fig. $6)$.

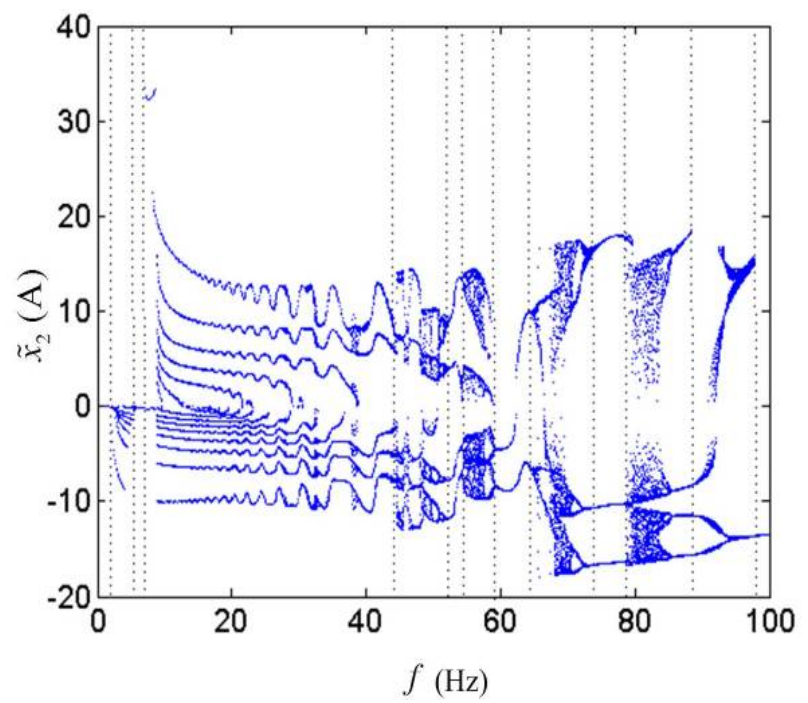

Fig. 2. Bifurcation diagram of the BLDCM

When the load is constant, that is, the frequency of load vibration $f=0 \mathrm{~Hz}$, the waveforms of the variable $\tilde{x}_{1}, \tilde{x}_{2}$, and $\tilde{x}_{3}$, and the phase trajectory between the three variables are observed, as exhibited in Fig. 3. The system state tends to stabilize after starting oscillation in the absence of torque disturbance. The phase trajectory of the system converges to a stable point, while the system is in a stable state.

When $f=20 \mathrm{~Hz}$, the phase trajectory between the three variables and the power spectrum of the q-axis current are observed, as displayed in Fig. 4. The period doubling loop occurs on the phase plane. Moreover, peaks are observed at several frequencies in the power spectrum of the q-axis current of the system. Therefore, the system has a periodic motion at the corresponding frequencies.

When $f=70 \mathrm{~Hz}$, the phase plane diagram of the system and the power spectrum of q-axis current are observed, as presented in Fig. 5. Singular attractors appear on the phase plane. The phase trajectory seems random, but it has a selfsimilar structure. Moreover, the power spectrum of the qaxis current exhibits periodicity. Thus, the system is in a chaotic motion.

When $f=98 \mathrm{~Hz}$, the phase plane and power spectrum of the q-axis current are observed, as illustrated in Fig. 6. The limit cycle appears in the phase trajectories. Simultaneously, only one peak appears in the power spectrum of the q-axis current. Thus, the phase trajectories eventually tends to a stable limit cycle and enters the uniperiodic motion.
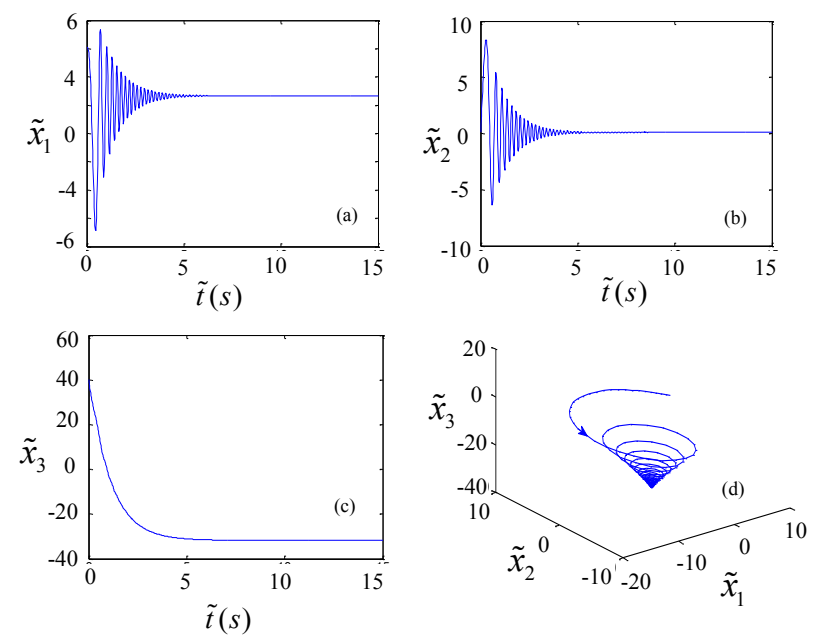

Fig. 3. $\tilde{x}_{1}, \tilde{x}_{2}, \tilde{x}_{3}$ and the phase trajectory $(f=0 H z)$
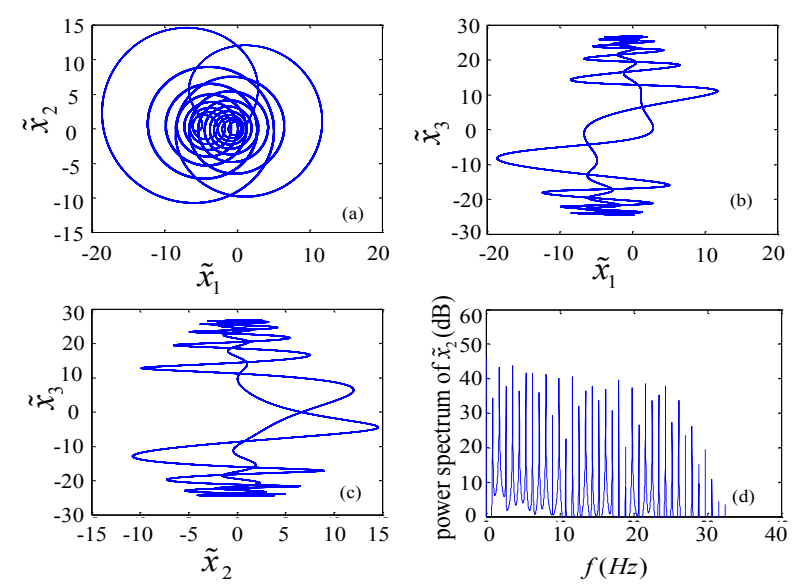

Fig. 4. Phase trajectory and power spectrum $(f=20 \mathrm{~Hz})$

Given that the frequency of the oscillating load varies, the BLDCM system presents complex non-linear dynamic characteristics, namely, equilibrium point, limit cycle, cycle motion, period doubling, and chaotic motion. By combining Fig. 1 and Fig. 2, we can conclude that, with the change in parameter, the systems generate period doubling motion ( $f$ $\in[6.6,43.9] \mathrm{Hz})$, paroxysmal chaos $(f \in[43.9,58.9] \mathrm{Hz})$, cycle motion $(f \in[58.9,65] \mathrm{Hz})$, paroxysmal chaos $(f \in$ $[65,84.2])$, and cycle motion sequentially.
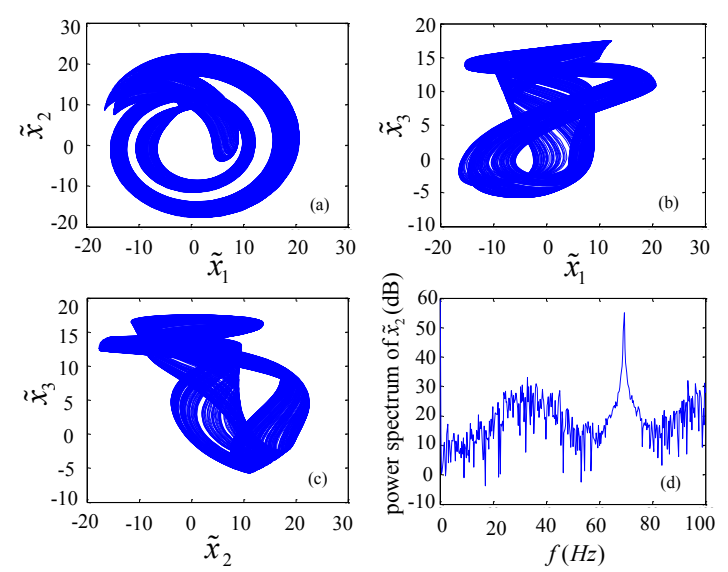

Fig. 5. Phase trajectory and power spectrum $(f=70 \mathrm{~Hz})$ 

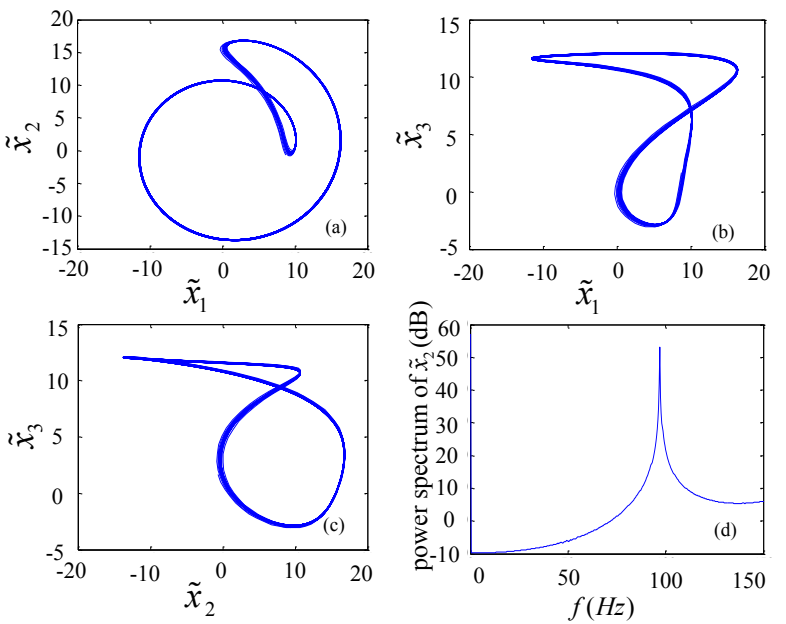

Fig. 6. Phase trajectory and power $\operatorname{spectrum}(f=98 \mathrm{~Hz})$

\subsection{Experimental studies}

In this study, the vibration load of the BLDCM is generated using a dynamometer.

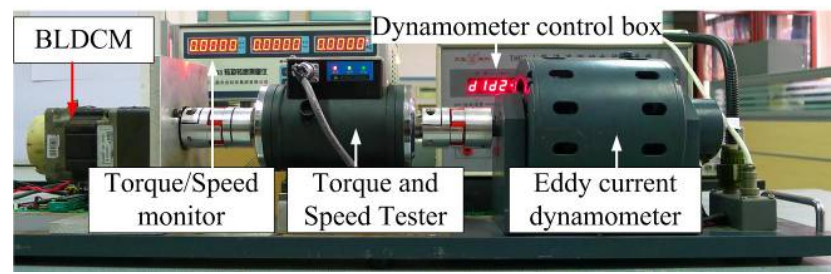

Fig. 7. Test platform of torque-current characteristic for an eddy current dynamometer

Fan [24] demonstrated that, under certain conditions, the braking torque of an eddy current dynamometer has an approximate linear relationship with excitation current. The characteristics of the dynamometer used in the experiment are tested. The experimental device is depicted in Fig. 7. The type of eddy current dynamometer used in the experiment is THCG-1, which is manufactured by Zhejiang Tianhuang Science and Technology Industry Co., Ltd. (power range is $0 \sim 1500 \mathrm{~W}$, torque range is $0 \sim 2 \mathrm{Nm}$, speed range is $1 \sim$ $3000 \mathrm{r} / \mathrm{min})$. The model of torque and speed measuring instrument used in the experiment is JN338, which is manufactured by Beijing Sanjing Pioneer Technology Group Co., Ltd.

After experimental testing, the relationship between braking torque and excitation current of the eddy current dynamometer is demonstrated in Fig. 8. When the speed of the dynamometer is between 1000 and 2000rpm and the excitation current varies between 0.4 and $0.7 \mathrm{~A}$, the braking torque varies approximately equally; that is, the braking torque is approximately proportional to the excitation current

To ensure that the rotational speed of the experimental platform is constant, the DC generator provides a large constant load torque in the experiment. Then, the eddy current dynamometer provides a small load disturbance torque. Thus, we can solve the problem in which the response speed of the BLDCM control system cannot keep up with the change in the torque when the load fluctuation is unreasonably large. We also achieve the purpose of the basic stability of the system speed. The torque of the DC generator is determined through its excitation voltage and armature load resistance. The related parameters can be calibrated through the torque and speed sensor in advance. The experimental platform is displayed in Fig. 9. The BLDCM drives the DC generator and eddy current dynamometer simultaneously through coupling. The parameters of the BLDCM are provided in Section 3.1.

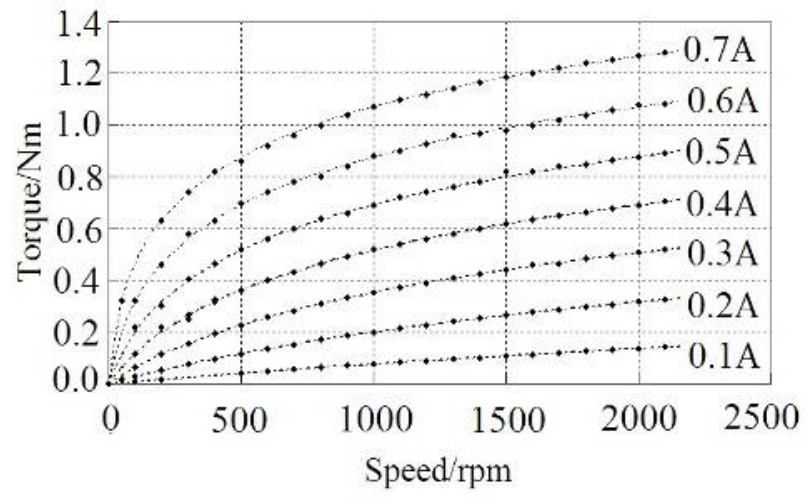

Fig. 8. Torque-current characteristic of a dynamometer

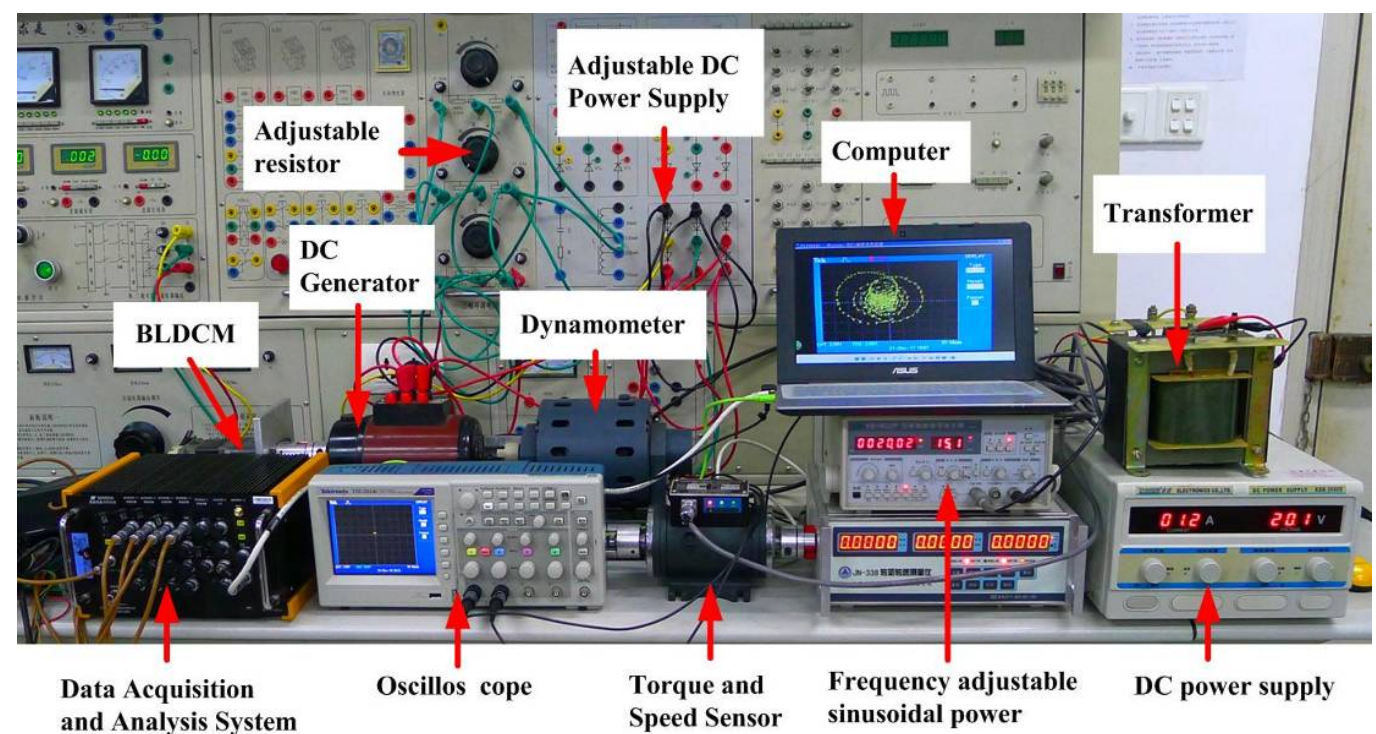

Fig. 9. Experimental platform for the nonlinear characteristics of the BLDCM 
In the experiment, the driving speed of the BLDCM is adjusted to $1500 \mathrm{rpm}$. By adjusting the excitation voltage and armature load resistance, the DC generator generates $3.5 \mathrm{Nm}$ load torque. By applying the AC/DC hybrid excitation current $i_{f}=0.6+0.5 \sin (\omega t) \mathrm{A}$ to the dynamometer, as presented in Fig. 8, the braking torque is approximately $T_{L}=1+0.8 \sin (\omega t) N m$. The superposition of the DC and AC power supplies is accomplished by a transformer and a capacitor.

In the experiment, the working current, rotational speed, and excitation current of the BLDCM dynamometer are collected through data acquisition and analysis system (DH5902). Then, they are displayed through calculation and conversion. The computer is equipped with oscilloscope (TDS2024C) simulation software produced by the Tektronix Company. It can replace the oscilloscope to display part of the data in real time and simplify the interface for observation. The frequency of the excitation power supply of the dynamometer is gradually changed. The phase trajectories of the system, current waveform, and power spectrum waveform are observed in the computer screen and oscilloscope. The operation characteristics of the motor are analyzed and compared with the theoretical analysis.

\section{Result Analysis and Discussion}

When the frequency of load vibration $f=0 \mathrm{~Hz}$, the time domain waveform of the q-axis current $i_{q}$ is illustrated in Fig. 10. In the interface of the oscilloscope, the longitudinal coordinate is the current amplitude, and the Abscissa is time variable. After conversion, the scale of current is $0.5 \mathrm{~A} / \mathrm{div}$, and the scale of time is $0.015 \mathrm{~s} / \mathrm{div}$. This study primarily focuses on the shape and trend of the waveform. Thus, it ignores the measurement and display errors of the instrument. When the motor starts, the q-axis current oscillates, and the current amplitude gradually decreases and stabilizes. The plane phase trajectory of the $\mathrm{d}-\mathrm{q}$ axis current of the motor is shown in Fig. 11. The phase trajectory converges slowly to the central stable point from the outer ring. It verifies that the system is in stable motion without the disturbance of load vibration.

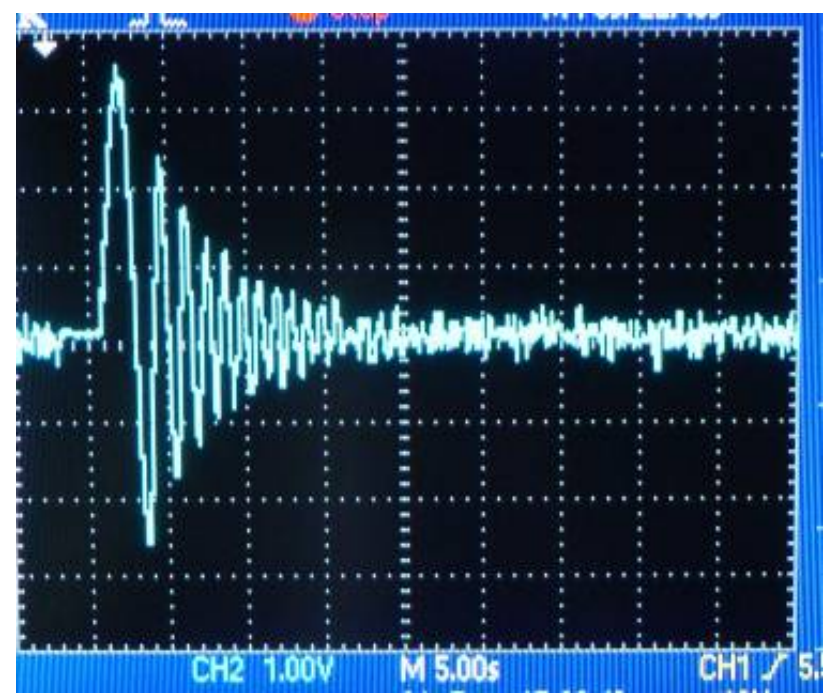

Fig. 10. Q-axis current $i_{q}$ at start-up ( $\left.f=0 \mathrm{~Hz}\right)$

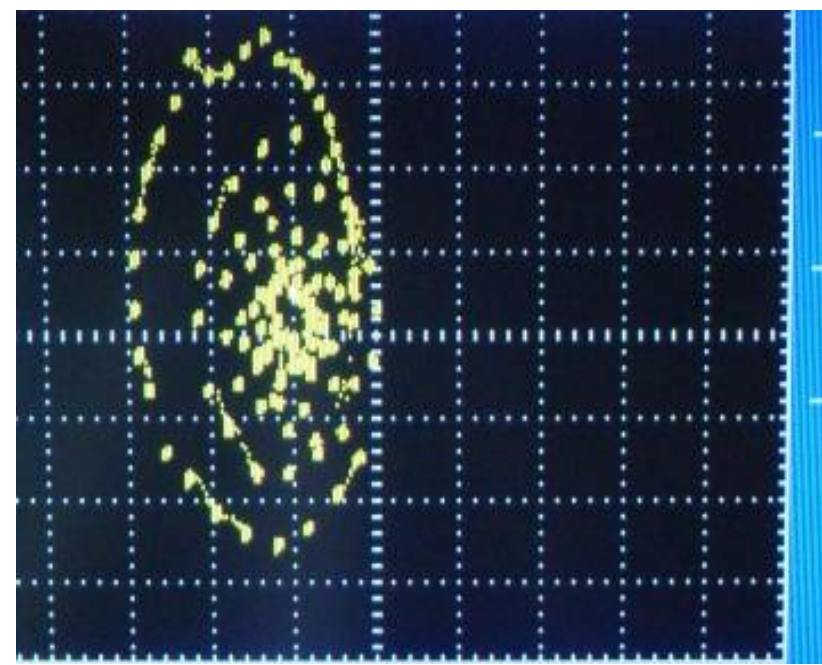

Fig. 11. Phase trajectory of $i_{d}-i_{q}(f=0 \mathrm{~Hz})$

When the vibration frequency of the torque equals 7.1 $\mathrm{Hz}$, the phase trajectory of the $\mathrm{d}-\mathrm{q}$ axis current of the motor is observed, as depicted in Fig. 12. The graph exhibits many circular trajectories that do not converge to the central stable point. Thus, the system is in the state of doubling periodic motion. Fig. 13 demonstrates the q-axis current and its power spectrum.

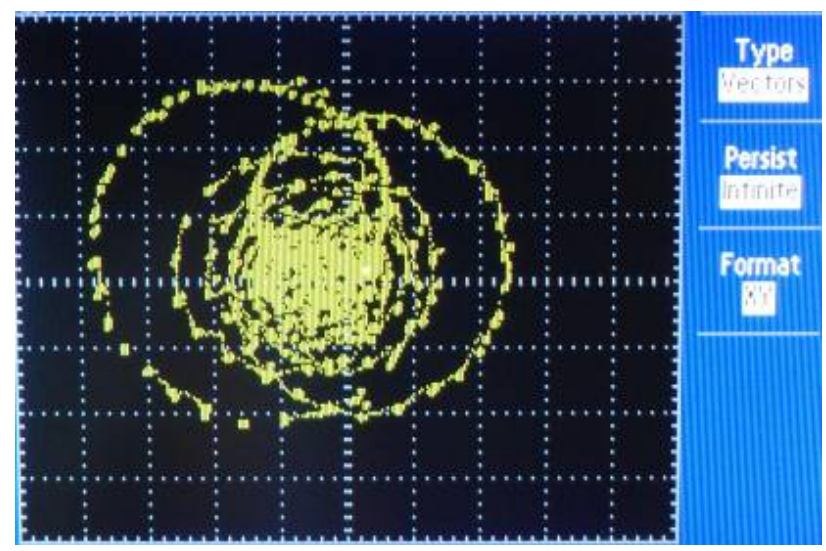

Fig.12. Phase trajectory of $i_{d}-i_{q}(f=7.1 H z)$

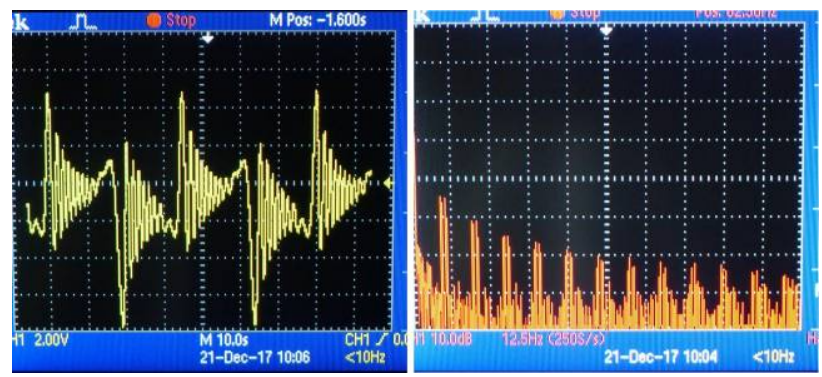

Fig. 13. Q-axis current and its power spectrum $(f=7.1 \mathrm{~Hz})$

The power spectrum contains many peaks. Thus, the system is in the state of doubling periodic motion. When the frequency is increased to $f=44.7 \mathrm{~Hz}$, the approximate shape of the phase trajectory and current power spectrum remains unchanged.

When the frequency of load vibration rises to $44.8 \mathrm{~Hz}$, the plane phase trajectory of the $\mathrm{d}-\mathrm{q}$ axis current is observed, as displayed in Fig. 14. 


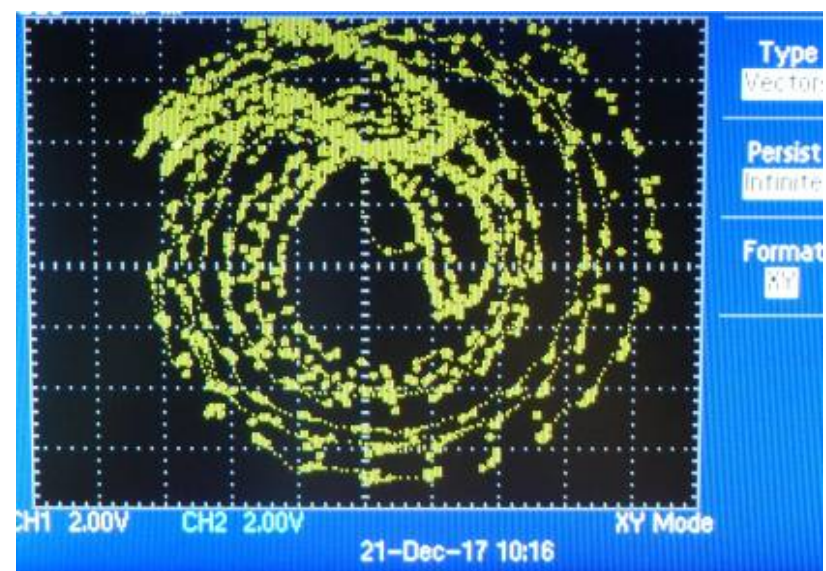

Fig.14. Phase trajectory of $i_{d}-i_{q}(f=44.8 H z)$

The phase plane trajectories are interlaced with each other. Moreover, no evident fixed orbit is observed. Fig. 15 illustrates the q-axis current and its power spectrum. The time domain waveform of the current presents no periodicity. Furthermore, the whole frequency domain of the power spectrum has numerous peaks, thereby indicating that the system has entered the chaotic state. By increasing the frequency of the vibration load, the approximate shape of the current and power spectrum is maintained at $52.2 \mathrm{~Hz}$.
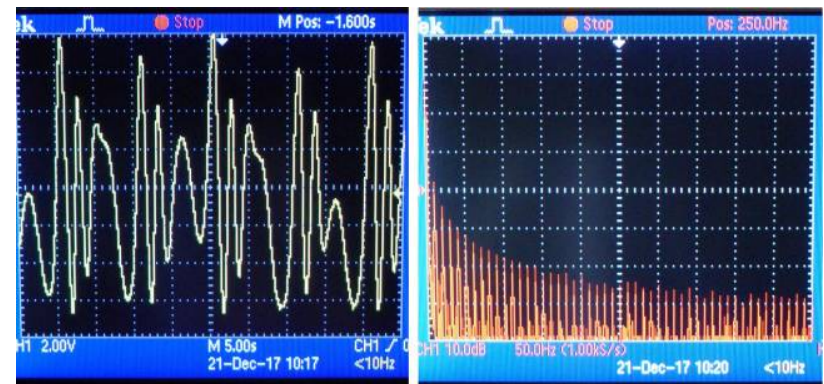

Fig. 15. Q-axis current and its power spectrum $(f=44.8 \mathrm{~Hz})$

Given that the frequency of load vibration continues to increase to $52.3 \mathrm{~Hz}$, the plane phase trajectory of the $\mathrm{d}-\mathrm{q}$ axis current presents a limit cycle, as depicted in Fig. 16. Fig.17 demonstrates the power spectrum of the d-axis current, which contains only one peak impact. Therefore, the system degenerates into a stable periodic one motion state. This state continues until the frequency rises to $54.5 \mathrm{~Hz}$.

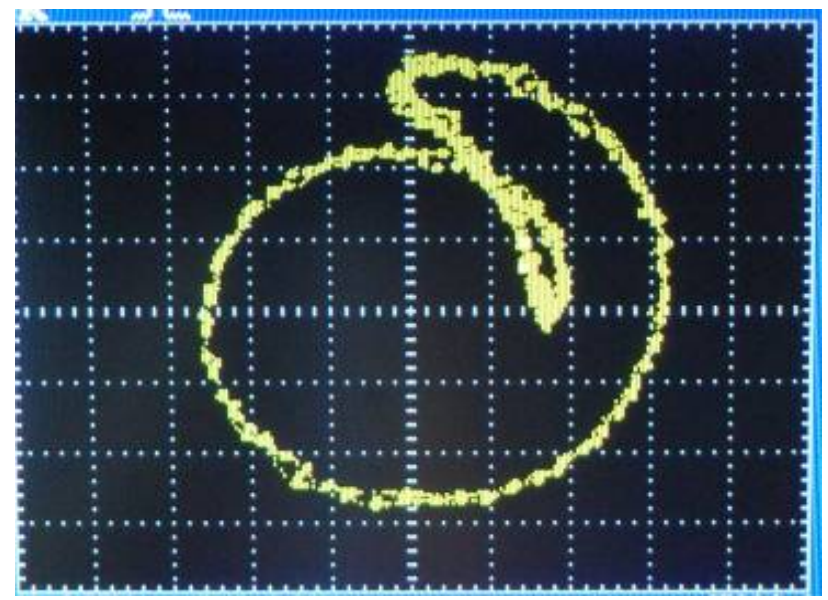

Fig.16. Phase trajectory of $i_{d}-i_{q}(f=52.3 \mathrm{~Hz})$
When the frequency rises to $54.6 \mathrm{~Hz}$, the waveforms of the phase plane and current power spectrum are similar to that in Fig.14 and Fig.15. Thus, the system is back to the chaotic state. The experimental results are consistent with the theoretical analysis results provided in Section 3. Several errors of frequency range between the experiment and the previous theoretical analysis are observed. These errors are mainly caused by the experimental errors and do not affect the experimental results.
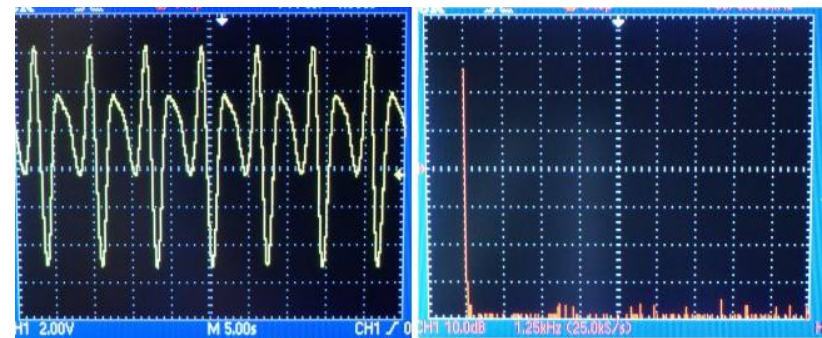

Fig. 17. Q-axis current and power spectrum $(f=52.3 \mathrm{~Hz})$

\section{Conclusions}

To explore the nonlinear dynamic characteristics of a BLDCM under load torque disturbance, we initially introduced the vibration load disturbance to build a 5-D mathematical model of the BLDCM by using time scale and linear affine transformations. Afterward, we analyzed and simulated the dynamic characteristics of the motor theoretically. Finally, we built the experimental platform to verify the results. Through theoretical analysis and experiments, the following conclusions were drawn.

1) With the change in the frequency of load vibration component, the BLDCM system presents the stable point, limit cycle, period, period doubling, and chaotic motion. When the frequency is between 6.6 and $43.9 \mathrm{~Hz}$, the system is in the period doubling motion. When the frequency is between 43.9 and $58.9 \mathrm{~Hz}$, the system enters the paroxysmal chaotic motion. When the frequency is between 58.9 and 65 $\mathrm{Hz}$, the system enters the periodic motion state.

2) When the frequency of the load vibration component continuously increases and the frequency is between 65 and $84.2 \mathrm{~Hz}$, the system enters the paroxysmal chaotic motion again. When the frequency continuously rises, the system enters the periodic motion state.

This study is helpful in preventing the torque and speed oscillation of the BLDCM drive system caused by vibration load. It also provides the goal and direction for searching for the optimal control of the motor system under vibration load disturbance. Therefore, this study has strong theoretical and practical significance because it primarily focuses on the nonlinear phenomena caused by the frequency change in sinusoidal vibration. However, it does not discuss the nonlinear phenomena caused by other waveform vibrations. This lack of discussion is the major limitation of this study. The non-linear characteristics of the BLDCM under the interference of other vibration waveforms and the corresponding control methods must be further studied in the future.

\section{Acknowledgements}

The study was supported by the Natural Science Foundation of Zhejiang Province of China (Grant No.LY17E050004) 
and Social Public Welfare Project of Zhejiang Science and Technology Department (Grant No. 2015C33242).
This is an Open Access article distributed under the terms of the Creative Commons Attribution Licence

\section{References}

1. Duqu Wei, Xiaoshu Luo, Bo Zhang, "Synchronization of brushless DC motors based on LaSalle invariance principle". Nonlinear, 69(4), 2012, pp.1733-1738.

2. Lin $\mathrm{Xu}, \mathrm{Y}$ un Chen,Zhong Liu, "Finite-time synchronization for the brushless DC motor systems". Control Theory \& Application, 31(1), 2014, pp. 78-84.

3. M. A. bin Abu Hassan, Bin Abdullah, A. R. bin Abdullah, "Performance Analysis of Brushless DC Motor Drive for Air Conditioner". Applied Mechanics and Materials, 785(8), 2015, pp. 243-247.

4. P. Rajasekaran, K. Vanchinathan, "Improved Performance of Four Switch Three Phase Brushless DC Motor using Speed-Current Control Algorithm". International Journal of Computer Applications, 68(11), 2013, pp.1-7.

5. R., shanmugasundram, K., Muhammad Zakariah, N., Yadaiah, "Implementation and Performance Analysis of Digital Controllers for Brushless DC Motor Drives". EEE/ASME Transactions on Mechatronics, 19(1), 2014, pp. 213-224.

6. Sukanya Parui, Biswarup Basak, "Nonlinear phenomena in permanent magnet Brushless DC motor drive". In: Michael Faraday Iet International Summit, New York, USA: Curran Associates, Inc., 2016, pp. 1617-1628.

7. Ping Zhou, Hao Cai, Chunde Yang, "Stabilization of the unstable equilibrium points of the fractional-order BLDCM chaotic system in the sense of Lyapunov by a single-state variable". Nonlinear dynamics, 84(2), 2016, pp. 2357-2361.

8. Fuchen Zhang, Da Lin, Min Xiao, "Dynamical behaviors of the chaotic Brushless DC motors model". Complexity, 21(4) , 2016, pp.79-85.

9. Weiya Zhang, Yongli Li, Xiaoyong Chang, "Dynamical investigation and parameter stability region analysis of a flywheel energy storage system in charging mode". Chinese Physics B, 22(9) 2013, pp. 619-632.

10. Shaohua Luo, Zhiwei Hou, Zhong Chen, "Chaos control for the output-constrained system by using adaptive dynamic surface technology and application to the brushless DC motor". AIP Advances, 5(12), 2015, 105-117.

11. Hemati, N., "Strange attractors in brushless dc motors". IEEE Transactions on Circuits and Systems I:Fundamental Theory and Applications, 41(1), 1994, pp. 40-45.

12. Akhgari Farzaneh, Rahmani Zahra, Rezaie Behrooz, "Anti-Control of Chaos of Single Time Scale Brushless DC Motor System with Unknown Parameters Using Adaptive Control". Mechatronics and Intelligent Materials, 211(12), 2011, pp. 736-741.
13. Mousam Ghosh, Goutam Kumar Panda, Pradip Kumar Saha, "Analysis of Chaos and Bifurcation Due to Slotting Effect and Commutation in a Current Discontinuous Permanent Magnet Brushed DC Motor Drive”. IEEE Trans.Industrial Electronics, 65(3), 2018, pp. 2001-2008.

14. Fadhil Rahma Tahir, Khalid M. Abdul-Hassan, Mohammed Abbas Abdullah, Viet-Thanh Pham, Thang Manh Hoang, Xiong Wang, "Analysis and Stabilization of Chaos in Permanent Magnet DC Motor Driver". World Scientific Publishing Company, 27(11), 2017, pp.1-14.

15. Jian Hu, Long Liu, Dawei Ma, "Robust nonlinear feedback control of a chaotic permanent-magnet synchronous motor with a load torque disturbance". Journal of the Korean Physical Society, 65(12), 2014, 2132-2139.

16. N. Hemati, M. C. Leu, "A complete model characterization of brushless DC motors". IEEE Transactions on Industry Applications, 28(1), 1992, pp. 172-180.

17. Weikang Qian, Yutao Shi, "Analysis and Simulation on Torque Ripples of Brushless DC Motor”. Telecommunication, computing, electronics and control, 13(2), 2015, pp. 381-390.

18. Yoni mandel, George Weiss, "Adaptive internal model based suppression of torque ripple in brushless DC motor drives". Systems Science \& Control Engineering, 3(1), 2015, pp.162-176.

19. Fengxia Tian, Fangchao Zhen, Guopeng Zhou, "New Results of Global Exponential Stabilization for BLDCMs System". Mathematical Problems in Engineering, 2015(5): 2015, pp.159167.

20. Esmat Sadat Alaviyan Shahri, Alireza Alfi, J. A. Tenreiro Machado, "Stability analysis of a class of nonlinear fractional order systems under control input saturation". International Journal of Robust and Nonlinear Control, 28(1) 2018, pp.2887-2905.

21. Yilmaz Uyaroglu, Baris Cevher, "Chaos control of single timescale brushless DC motor with sliding mode control method". Turkish Journal of Electrical Engineering \& Computer Sciences, 20(21), 2013, pp. 649-655.

22. Z. M. Ge, C. M. Chang, "Chaos synchronization and parameters identification of single time scale brushless DC motors". Chaos, Solitons and fractals, 20(6), 2004, pp. 883-903.

23. Zengqiang Chen, Zhuzhi Yuan, Shijian Cang, "Analysis and circuit implementation of a new four-dimensional non-autonomous hyperchaotic system". Journal of Physics, 57(3): 2008, pp. 1493-1501.

24. Shuang, F., "Development of Loading Control System for Eddy Current Dynamometer". Master thesis of Beijing University of Technology, China, 2016, pp.21-28. 